\title{
La educación en el siglo XXI: Una mirada incipiente desde las neurociencias al quehacer pedagógico
}

\author{
Lía Anchía-Angulo * \\ https://orcid.org/0000-0002-5381-8768 \\ Tobías Brizuela-Gutiérrez ** \\ https://orcid.org/0000-0001-7291-0634
}

\section{Resumen}

Por ser la educación el objeto de estudio de la Administración Educativa, constituye una prioridad continua de la administración repensarla. Esa tarea, actualmente, comprende demarcar su rol en la sociedad de la información y el conocimiento, el abordaje más propicio para el desarrollo de las habilidades que esta demanda.

En Costa Rica, este hecho, inexorablemente implica volver la mirada hacia las personas que le dieron un rostro social y cultural a la educación. Entre ellas, María Isabel Carvajal Castro (más conocida como Carmen Lyra), quien proponía la intrínseca relación entre la enseñanza y la construcción de la persona crítica ${ }^{1}$.

En el contexto del bicentenario, el siguiente ensayo ${ }^{2}$ tiene como objetivo central puntualizar, de manera crítica y reflexiva, prácticas que el sistema educativo costarricense requiere superar para responder a una educación de calidad. El Séptimo Informe del Estado de la Educación ${ }^{3}$ revela que, si bien la intención del Estado apunta a la mejora, no se están alcanzando indicadores satisfactorios.

Con el fin de responder a esa transformación educativa, el artículo intenta sustentar su argumento central con los aportes de neurociencias que, aunque de modo tenue aún, permean el ámbito de la educación costarricense. Un amplio abanico que la dota de múltiples posibilidades de optimizar el proceso de enseñanza-aprendizaje para así alejarse de las ya devaluadas prácticas del sistema tradicional y acceder más y mejor a niños y jóvenes.

Palabras clave: Calidad educativa, gestión, pedagogía.

\footnotetext{
${ }^{1}$ Maurizia D' Antoni, «Carmen Lyra: una Pedagogía acallada en Costa Rica y sus vinculaciones teóricas» (Ponencia del $3^{\text {er }}$ Congreso Internacional de Investigación Educativa. Educación y Globalización. Instituto de Investigación en Educación, Universidad de Costa Rica, 2015).

${ }^{2}$ Aprobado por el comité editorial de la Revista Espiga en sesión del 14 de mayo de 2021. La sección en que se publica no es arbitrada mediante el proceso de pares.

${ }^{3}$ Programa Estado de la Nación en Desarrollo Humano Sostenible, Resumen Séptimo Informe Estado de la Educación (San José: Masterlitto, 2019).
} 
* Estudios en Filología Española, Facultad de Filología Romano-Germánica de Odessa, Ucrania. Bachiller en Ciencias de la Educación con Énfasis en I y II Ciclos, Universidad Latina de Costa Rica. Máster en Trastornos del Aprendizaje con Mención Neurológica, Universidad Americana. Máster en Administración Educativa, Universidad de Costa Rica. Diplomado en Neuropsicoeducación, Universidad de Buenos Aires, Argentina. Diplomado Universitario de Capacitación Docente en Neurociencias, Universidad Abierta Interamericana UAI, Buenos Aires, Argentina. Directora Académica del Colegio Internacional Canadiense. Correo: lia.anchia@gmail.com

** Master en Educación con Énfasis en la Enseñanza del Inglés, Universidad Latina de Costa Rica. Licenciado en Educación con Énfasis en Currículo y Evaluación, Universidad de las Ciencias y el Arte. Bachiller en la Enseñanza del Inglés para I y II ciclos, Universidad Estatal a Distancia (UNED). Encargado de la Cátedra de Enseñanza del Inglés de la Escuela de Ciencias Sociales y Humanidades, de la UNED. Profesor titular del área de Enseñanza del Inglés y docente de grado de asignaturas relacionadas con la carrera de Enseñanza del Inglés para I y II ciclos, de la UNED. Correo: tbrizuela@uned.ac.cr 


\section{Introducción}

El campo de la educación es uno de los más complejos y demandantes; es una realidad que, por una parte, es irremediablemente débil mientras que por otra es extremadamente fuerte. En medio de ese nado en dos aguas, época tras época, la sociedad continúa apostando por ella para que resuelva los retos y desafíos que afloran a su paso, se le sigue delegando la preservación de la especie y su acervo. Ya indicaba Rigoberta Menchú, premio Nobel de la Paz, que «La educación es alma del desarrollo de los pueblos» ${ }^{4}$.

Actualmente, en algunos escenarios más intencionadamente que en otros, se transita de un modelo educativo basado en la «patología»-que aún lleva a muchos profesionales y «especialistas» a ejercer la profesión centrados en detectar el defecto o el error- a uno que más bien resalta todo lo enriquecedor que es el error para el propio proceso de aprendizaje. El primer enfoque, centrado en «castigar el error», desemboca en dos situaciones igual de lacerantes: por un lado, hay docentes que apenas sobreviven a un sistema que patologiza y, por otro, a estudiantes cuyo único mensaje recibido es «ustedes son incapaces». La continuación de este ciclo ocurre cuando, en ese recorrido escolar, una buena parte de estudiantes se creen esa sentencia y llegan al sistema con el intento de demostrar, a toda costa, que «son capaces». Entonces, gracias a los renovados aires que soplan en el sistema educativo, se estaría a las puertas de la reivindicación del error, concebido ahora como uno que juega en favor del aprendizaje y no en contra; más aún, en una de sus principales fuentes.

No es posible resignificar la educación de Costa Rica sin volver la mirada agradecida hacia sus propulsores, para no desligarse de la acción transformadora que ellos le atribuían desde entonces. María Isabel Carvajal Castro, más conocida como Carmen Lyra, proponía la estrecha relación entre la enseñanza y la construcción de la persona crítica, privilegiando como función de la formación aquella de crear personas que tengan motivación hacia el aprendizaje, que sepan relacionar las nociones entre sí y cómo y dónde buscar más ${ }^{5}$. Ella conjugaba con las ideas de Jerome Seymour Bruner, para quien la construcción del conocimiento se logra mediante la inmersión del estudiante en situaciones de aprendizaje problemáticas, concebidas para retar la capacidad del aprendiz en la resolución de problemas diseñados de tal forma que el estudiante aprenda descubriendo ${ }^{6}$. Con este propósito humanizante, Bruner, precisamente, concebía la institución educativa como el espacio propicio para el desarrollo integral de la persona, uno ampliamente disponible para el estudiante, uno que no se circunscribía nada más al aula ni mucho menos al despliegue de contenidos curriculares.

La actual sociedad se caracteriza por un desarrollo vertiginoso en el ámbito de la información y la comunicación, facilitado por los grandes avances tecnológicos. No solamente se han acortado las distancias en términos de acceso a la información, sino en lo que respecta a la producción y acceso al conocimiento. El fenómeno

\footnotetext{
${ }^{4}$ César Pérez, «La educación es alma del desarrollo de los pueblos, dijo Rigoberta Menchú», 16 de febrero de 2011, acceso: 31 de agosto de 2021https://www.lavozdelsandinismo.com/nicaragua/201102-16/la-educacion-es-alma-del-desarrollo-de-los-pueblos-dijo-rigoberto-menchu/

${ }^{5}$ D’ Antoni, «Carmen Lyra: una...

${ }^{6}$ Bibliotecas blog, «J. S. Bruner y su aportación a la Educación», 1 de octubre de 2015, acceso: 31 de agosto de 2021, http://blogbibliotecas.mecd.gob.es/2015/10/01/j-s-bruner-y-su-aportacion-a-laeducacion/
} 
informacional ha generado cambios sustanciales en el ritmo de vida de las personas, en su capacidad de respuesta y, muy probablemente, en su manera de concebir el mundo. Pero, por diversas razones, la adaptación al cambio no ocurre con la misma inmediatez que estos emergen: apenas las personas han asimilado algunos, vienen otros a colocarse en la palestra.

\section{¿De qué modo responder a esos retos y desafíos actuales en educación?}

Un aforismo atribuido a Confucio reza: «Los pueblos que no conocen su historia están condenados a repetirla». Esta máxima no solamente es aplicable a los pueblos, también a las personas y a las organizaciones. Las personas pocas veces se detienen a pensar acerca del origen de una respuesta, de cualquier respuesta, ya sea que se le dé a un hijo, estudiante, colaborador, compañero de trabajo u otra persona.

El Sétimo Informe del Estado de la Educación advierte que «Es indispensable reorganizar los servicios educativos en primaria para que, a corto plazo, la mayoría de los centros impartan el currículo completo, con los contenidos y pedagogía aprobados por el Consejo Superior de Educación» ${ }^{7}$. Se está frente a dos grandes temas: la cobertura y la calidad, aunque también se podría citar un tercero, el actual modelo de gestión. Y ninguno es excluyente. De hecho, este mismo informe señala que no han sido pocos los intentos de los costarricenses por mejorar su sistema educativo, sin embargo, también apunta a que, pese a todos los esfuerzos, todavía no se logra salir de la «jaula» estructural de la escuela per se y, paralelamente, de la gestión. ${ }^{8}$ Sin duda, este planteamiento del Informe de la Educación pone a pensar en las posibles repercusiones que tiene para la sociedad costarricense el alcanzar o no una educación de calidad.

Si bien cada año se puntualizan avances, todavía es amplio el camino por recorrer para que la educación responda a las demandas del siglo XXI. Para Ortiz,

El fracaso del siglo pasado en el ámbito formativo fue la educación. A pesar de los avances en las ciencias pedagógicas y en la didáctica, las organizaciones educativas aún siguen utilizando un entumecido y añejo sistema para comunicar conocimientos, que por cierto ya ha sido devaluado por ser obsoleto. ${ }^{9}$

El artículo 1 de la Ley fundamental de Educación indica que «Todo habitante de la República tiene derecho a la educación y el Estado la obligación de procurar ofrecerla en la forma más amplia y adecuada». Sin embargo, algunos de los alcances del último informe del Estado de la Educación (2019), concretamente el «segundo mensaje» arrojado por las investigaciones que sustentan dicho informe, referente a los logros en educación, devela que la intención del Estado de universalizar la educación de calidad no necesariamente está alcanzando indicadores satisfactorios. Advierte sobre la seriedad de los factores que le impiden a un estudiante vivir una experiencia educativa de calidad ${ }^{10}$. Al respecto, agregan que es crucial modificar el modo de selección o contratación docente, dado que el actual se considera obsoleto. Por otra parte, no solamente señalan las debilidades que tienen algunos de los docentes contratados, sino que las capacitaciones que ofrece el ente rector (el

\footnotetext{
${ }^{7}$ Programa Estado de la Nación en Desarrollo Humano Sostenible, Resumen Séptimo Informe..., 16.

${ }^{8}$ Ibíd., 20.

${ }^{9}$ Alexander Ortiz Ocaña, Neuroeducación. ¿Cómo aprenden los estudiantes y cómo deberían enseñar los docentes? (Bogotá: Ediciones de la U, 2015).

${ }^{10}$ Programa Estado de la Nación en Desarrollo Humano Sostenible, Resumen Séptimo Informe..., 16.
} 
Ministerio de Educación Pública, MEP) no son mecanismos efectivos para corregir tales deficiencias en la formación.

A partir de las premisas anteriores, este artículo es un intento por esbozar, aunque de manera laxa aún, algunas de las contribuciones que le devuelve las neurociencias a la educación. Se afirma así porque el trabajo de investigación no es lineal, va y viene, incluso, en muchas direcciones. Y no es dicotómico, toma algo del ámbito propio y le devuelve algo también.

Con base en esos insumos, es imperativo aprender a mirar a los estudiantes a través de un nuevo prisma, aprender a mirarlos como si no se les hubiera mirado nunca, debe interesar más la persona, debe importar más educar, en el amplio sentido de esa palabra. Los estudiantes, para salir del ciclo al que los condena un sistema basado en la detección de la «patología»-el error visto como falla nada más, la persona fusionada con ese error y asumiéndose como si fuera el error, el número-, tal y como apuntan los neuroinvestigadores, requieren experimentar el éxito, contar con pruebas palpables de que pueden obtener logro, vivir el logro, sentir que son personas y no un medio, no un número. "A los educadores nos preocupa que la patologización precipitada de algunas conductas nos impida ver las posibilidades de esos niños», refiere con contundencia Marina ${ }^{11}$. Un modelo educativo que respete verdaderamente la diferencia y sea incluyente debe migrar de los contenidos y refugiarse en los «centros de interés» de las personas, darles voz y voto (uno de los principales nutrientes de la motivación intrínseca). Para Frith y Blakemore, «(...) la educación puede ser considerada como una especie de 'jardinamiento' del cerebro, por tanto, los docentes sus jardineros» ${ }^{12}$. Y los buenos jardineros conocen la variedad y calidad del «abono» que emplean según sea la planta que cuidan, lo que sería análogo en el quehacer pedagógico, al conocimiento y dominio ya no del «qué» (tan abundante en la era de la información y del conocimiento), sino del «cómo». La pregunta orientadora entonces es «¿Cómo lo hacemos?». Esto conlleva, ineludiblemente, a pensar, repensar, significar y resignificar el acto de educar y, por ende, la gestión de la educación.

En América Latina, a lo largo del siglo XX, se implementaron diversos modelos educativos de bases filosóficas y administrativas distintas, en respuesta a las condiciones de la época. Lo anterior abarca la transición de la economía de base agrícola a la industrial, por ejemplo. Y si bien no podría detenerse en esto, sí es relevante comprender que, en la década de 1980, ocurrieron cambios sustanciales y estos también tuvieron a la calidad de la educación, como en casi todas las épocas, en la mesa de discusión. Con ella, también al modelo educativo, más enfocado en la conducta de la persona, al que más tarde le sucedería uno más centrado en el potencial de esta, como es el caso del enfoque constructivista.

Sin perder de vista que ya se ha caminado un par de décadas del nuevo siglo, todavía se puede afirmar que la gestión es una disciplina relativamente reciente, data de la década de 1960 en Estados Unidos, de los 70 en el Reino Unido y de los 80 en América Latina ${ }^{13}$. Valga el juego de palabras para enfatizar que se trata, quizá, de una disciplina en gestación constante. Además, está influenciada por un marco

\footnotetext{
${ }^{11}$ José Antonio Marina, «Neurociencia y educación. Participación Educativa», Revista del Consejo Escolar del Estado 1, n. ${ }^{\circ} 1$ (2012): 1-13.

${ }^{12}$ Sarah Blakemore y Uta Frith, Cómo aprende el cerebro. Las claves para la educación (Barcelona: Editorial Ariel, 2011).

${ }^{13}$ Juan Cassasus, «Problemas de la Gestión. La tensión entre los paradigmas A y B», Revista Brasilia, 19, . $^{\circ} 75$ (2002), $49-69$.
} 
político, económico, social, legal. Aquí cabe mencionar, entre otros, la Ley Fundamental de Educación, los pilares de esta, las políticas educativas.

\section{Gestión}

Gestión, una palabra que llega al entorno educativo con un matiz movilizador, horizontal y poco lineal, viene a romper con la tendencia verticalista, centralizada y autoritaria de la década de 1980, cuando se bifurcaba en una práctica que apuntaba a la planificación y otra a la administración. Los intentos por alcanzar la descentralización han venido a superar esa práctica, cambiando las competencias de los involucrados. Un proceso que se ha venido viviendo en la realidad actual mediante la implementación de modelos de gestión educativa conocidos como Gestión 10 y, más recientemente, Gestión 15.

De lo expuesto se desprende que, de quien gestiona se espera algo más que una dirección. El gestor educativo debe saber planificar y saber administrar. De ahí que una de las situaciones que más obstruyen la gestión es la inflexibilidad con que se lleva a cabo, pues, tal y como lo expone Casassus, «En la práctica el plan es solo una orientación y no una instrucción para ejecutarlo» ${ }^{14}$. Por consiguiente, gestionar implica contar con un pensamiento flexible, pues las organizaciones están inmersas en sistemas o son un subsistema de otros sistemas, así que se requerirá de la adaptación y del replanteamiento de esas orientaciones al contexto que se ocupa. Es decir, en medio de procesos y subprocesos, se pone a prueba, como señala este mismo autor, el talento político para lograrlo. La adaptabilidad, por tanto, es fundamental, requiere trabajar sin rigidez; con guías, sí, pero sin que estas se conviertan en estructuras inflexibles que solo obstruyen y complican el camino.

Se está frente a una de las mayores oportunidades y urgencias de mejora en lo que a gestión respecta y, por consiguiente, una mejora en la calidad de la educación del siglo XXI. Uno de los hallazgos del Séptimo Informe del Estado de la Educación indica que en el MEP

(...) urgen nuevos estilos de gestión que cambien prácticas y sentidos de acción, considerando las prioridades de asesoramiento e intervención centradas en lo pedagógico. Si no se modifica esta estructura, logros relevantes como las reformas curriculares y las nuevas estrategias, como las pruebas FARO o la ampliación de la educación dual, tienen baja probabilidad de éxito $(\ldots)^{15}$

En este contexto, una de las mayores dificultades es que, precisamente, se viven las consecuencias de la educación generada, «... la educación es a la vez producto de la sociedad y productora de esa misma sociedad», indica Carreño ${ }^{16}$, en buena medida, la sociedad actual se conduce con estricto apego a protocolos. Cuando quienes gestionan una institución educativa consideran que los protocolos son inamovibles, las acciones que promueven cobran un carácter rígido, pasivo, casi como siguiendo unas «indicaciones» que «bajaron» de los superiores jerárquicos. Lo anterior, en palabras del exministro de Educación durante los periodos 2006-2010 y 2010-2014, Leonardo Garnier Rímolo ${ }^{17}$, significa que no se asumen riesgos: ¡no se arriesgan!

\footnotetext{
${ }^{14}$ Ibíd., 101.

${ }^{15}$ CONARE (2019), 27.

${ }^{16}$ Ortiz Ocaña, Neuroeducación. ¿Cómo aprenden...

${ }^{17}$ Leonardo Garnier, «¿Mejor, no se arriesgue?», acceso: 31 de agosto de 2021,

http://leonardogarnier.com/articles/mep-subversivo/mejor-no-se-arriesgue-746
} 
El matiz negativo en esta constante es que se afecta a muchas personas, porque, como se mencionó anteriormente, los centros educativos son parte de un sistema dinámico. Esto se complica cuando, invadidos por la creencia de que el proceso de gestión es rígido, se omite que la razón de ser de la gestión educativa son los estudiantes y se proponen acciones importadas, acríticas, descontextualizadas que interfieren con lo que requieren experimentar personas en plena construcción. De ahí que, para que en lugar de despojar al estudiante de las expectativas que tenía previamente a su ingreso a la institución y más bien las aproveche para revitalizar continuamente los procesos, la gestión deba, por un lado, recubrirse de creatividad y alejarse de la «receta» y, por otro, actuar en consecuencia con el ineludible hecho de que «(...) la gestión es trabajar con personas» ${ }^{18}$.

La relevancia de comprender lo anterior estriba, en parte, en que previene que en educación se caiga en la confusión entre gestión y administración: se administran las cosas, los recursos, pero se gestionan las personas y los procesos. Implica entonces algo tan básico como «(...) la comprensión de lo que son las personas», para evitar caer en la cosificación de ellas y en la personificación de las cosas ${ }^{19}$. La persona, sea estudiante o colaborador de la institución educativa, debe sentirse persona, no un número, no un objeto ni mucho menos un medio para conseguir un fin.

De lo anterior, se podría deducir que los modelos de gestión apuntan a mejorar el futuro desde la acción, en ese intento se han probado distintos modelos que van desde el normativo hasta el de reingeniería y el comunicacional. Unos y otros han encontrado que el presente de cada época se contiene en un contexto histórico cultural al que debe responder con acciones en sintonía, congruentes. Sin duda que los niños del siglo XXI no pueden ser comprendidos a partir de la realidad familiar y social que vivía el niño de la década de 1980. Asimismo, los insumos que nutren a la educación actualmente, y que son operacionalizados por la gestión, no pueden responder a la comodidad de quienes enseñaron o se educaron en el siglo pasado.

En este sentido se hace imperante, por un lado, la necesidad inmediata de una revisión de las prácticas pedagógicas que están siendo implementadas por los que están al frente del acto educativo y, por otro, tal y como lo puntualiza el Informe del Estado de la Educación, una revisión de los procesos de contratación de docentes con el fin de seleccionar a los mejores candidatos a la docencia ${ }^{20}$. La realidad en el sistema educativo costarricense, público y privado, es que algunos de sus docentes requieren solventar vacíos en sus perfiles profesionales.

El origen de esta situación puede ser variado; no obstante, lo que se lee en el Informe del Estado de la Educación (2019) es que, independientemente de la titulación, la práctica pedagógica evidencia oportunidades de mejora en las áreas metodológicas, de planeamiento y diseño de materiales didácticos. No menos importante es que se promueva una efectiva correspondencia entre los contenidos, objetivos, las actividades, la evaluación de los aprendizajes propuestos y el estilo de gestión del aula. De igual modo, el uso de las tecnologías no debiera exceder su objetivo: ser un complemento en la mediación pedagógica, un instrumento que sirve para que el estudiante consolide su aprendizaje; lo relevante es que puedan usar lo aprendido en el contexto, por ende, no es recomendable que sustituya al docente ni que borre la

\footnotetext{
${ }^{18}$ Programa Estado de la Nación en Desarrollo Humano Sostenible, Resumen Séptimo Informe...

${ }^{19}$ Cassasus, Problemas de la...

${ }^{20}$ Programa Estado de la Nación en Desarrollo Humano Sostenible, Resumen Séptimo Informe..., 27. 
relevancia que tiene para un estudiante la toma de notas o la producción de resúmenes con sus propias palabras, por ejemplo.

La implementación de estrategias efectivas para responder a los estudiantes con necesidades educativas especiales vendría a ser un ingrediente imprescindible en la práctica docente. Comprendiendo que, muchas veces, el apego a protocolos lentificaría el despliegue de una oferta acorde con cada situación, las nuevas propuestas deberán acercar a los estudiantes al aprendizaje cooperativo, porque este abordaje amplía las posibilidades para todos. No se trata de borrar la individualidad, sino de todo lo contrario, de enriquecerla a través de la interacción.

Por otra parte, la supervisión de la labor docente en su campo de acción también requiere de un ligero matiz. Cuando los supervisores visitan los centros educativos, entre otros, deben poner a disposición de los gestores la información y los datos que les reflejen el funcionamiento y la actuación que está teniendo este. Además, abrir espacios para el debate de los problemas o necesidades que tenga el centro educativo, así como para la recepción de iniciativas en torno al mejoramiento de este. El curso de los debates no debe tener como objetivo el someter ni perseguir a nadie, sino el establecimiento de consensos y mecanismos de acción futura.

Para German Retana ${ }^{21}$

El término supervisar hace pensar en alguien que está ahí para verificar que los otros hagan lo que deben hacer, lo cual es un rol algo pasivo. Si cambiáramos ese enfoque por ser 'generador de equipo', entonces la connotación se volvería más productiva, educativa, orientadora y desarrolladora del talento y del alineamiento de comportamientos $\mathrm{o}$ desempeños. ${ }^{22}$

Desde esa perspectiva, lo que justifica la acción supervisora es asegurar la calidad de los procesos que se llevan a cabo en el interior de las instituciones educativas. Dejando atrás el sentido que tenía de recaudar una evidencia o señalar el error; ahora, sus acciones viabilizarían la mejora continua, lo cual debe estar siempre articulado con la evaluación de los procesos, empezando por la autoevaluación. Cuando se prescinde de la evaluación o de la valoración de los procesos, peor aún, cuando se cae en la justificación de los errores, se priva a todo un sistema de crecer, desarrollarse y alcanzar la consecución de la tarea común: una educación de elevado nivel de calidad. De modo que la actualización continua por parte de los gestores educativos se vuelve aquí una necesidad prioritaria y urgente. Sin evaluación, no hay mejora posible.

\section{Neurociencias en el aula: sus implicaciones}

Siguiendo un orden de ideas y el intento por vislumbrar opciones en esta hoja de ruta, sin ahondar más allá de lo que para estos efectos conviene, se puede afirmar que los procesos del desarrollo cognitivo siguen un orden, por lo que gestionar a los niños de la actualidad tampoco implica, como erróneamente se ha presupuesto, suprimir del todo actividades cruciales para atención oportuna de las distintas etapas de su desarrollo. Se hace referencia a aspectos tan básicos que han sido considerados apoyos «de más», tales como permitirles contar con los dedos, verbalizar la acción

\footnotetext{
${ }^{21} \mathrm{El}$ autor es $\mathrm{Ph} \mathrm{D}$, sociólogo, asesor comercial, conferencista nacional e internacional, creador y promotor del programa Gerencia con Liderazgo en organizaciones y equipos.

${ }^{22}$ German Retana, comunicación personal, 26 de abril de 2016.
} 
mientras resuelven un ejercicio, escribir en un cuaderno sus apreciaciones o plasmar sus emociones a través de un dibujo.

No menos frecuente es encontrarse que ahora se haya descuidado el estudio de los «gestos» (rasgos) de los grafemas, que es requisito indispensable para la adecuada inducción de la enseñanza de la lectoescritura: «El gesto de escritura tiene más virtudes. Orienta al niño en el espacio, ayudándolo a comprender que la cadena de letras debe leerse de izquierda a derecha. El reconocimiento del gesto también desempeña un papel esencial en el desciframiento de la escritura manuscrita» ${ }^{23}$. Previamente al estudio de los gestos, se debe trabajar entonces los trazos rectos, inclinados, curvos, que son prerrequisitos de estos otros, una práctica que ha caído en desuso como si el niño ya pudiera prescindir de estas etapas. Cuando se incurre en estas prácticas, se condena a muchos niños a tener que dibujar las letras en lugar que las puedan escribir con comprensión de la direccionalidad del trazo. Irremisiblemente, esto los aleja de vivenciar el placer a la hora de ir a la clase que tanto habían ansiado, esa donde le enseñarían a leer y escribir. Si se pregunta cuándo se desea repetir un aprendizaje, sin duda la respuesta a esta interrogante está en el aire: se desea repetir una experiencia de aprendizaje cuando esta ha sido placentera, a cualquier edad.

Dicho de otro modo, cuando la actividad académica es aplanada (sin emociones), difícilmente se quiere repetir. El ser humano tiene un instinto de supervivencia que se liga a una tendencia a buscar la felicidad, de modo que cuando hay emoción unida a la experiencia, la memoria que se crea es de las más duraderas. Lejos de subestimarlas para privilegiar otras de índole más académico, las áreas del desarrollo psicomotriz, socioafectiva y cognitiva son tres puertas imprescindibles de atravesar para contribuir al adecuado avance de los estudiantes en su experiencia educativa.

Siendo que la educación siempre ha sido enriquecida por distintas disciplinas, sin perder de vista que también las nutre a ellas, sobresale la urgencia de profundizar en lo que sea tendencia para «abrir la puerta» correcta en el momento oportuno.

(...) las investigaciones realizadas en el campo de la neurociencia vinculadas al aprendizaje, la memoria, las emociones, la motivación, los sistemas sensoriales, atencionales y motores, entre otros, indican que las propuestas curriculares deben incluir estos descubrimientos, ya que estos son de vital importancia para el campo educativo». ${ }^{24}$

Se trata, entonces, más de colocar los contenidos y los diferentes momentos que discurren en el centro educativo al servicio del despliegue del desarrollo integral de la persona, lo cual pone de relieve que a las habilidades complejas y abstractas -el control inhibitorio, la memoria de trabajo, la flexibilidad cognitiva, la metacognición, entre otras funciones ejecutivas- se llega pasando por la puesta en marcha de otras más sencillas y concretas, como son el seguimiento de patrones, los juegos dirigidos al aire libre, los juegos de mesa y otras que el docente promoverá de modo consciente y sistemático.

Desde el 2011, el Tercer Informe del Estado de la Educación revelaba la urgencia de que en el nivel de preescolar se implementaran actividades que propiciaran el desarrollo de algunas de las funciones ejecutivas (FE): control inhibitorio, memoria

${ }^{23}$ Dehaene Stanislas, Aprender a leer. De las ciencias cognitivas al aula (Buenos Aires, Argentina: Siglo Veintiuno, 2015).

${ }^{24}$ Programa Oficial de estudios para Preescolar, MEP, 11. 
de trabajo y atención. Con esta finalidad, los contenidos deben concebirse un medio y no un fin, un punto de partida para el desarrollo de estas y otras habilidades. Lo contrario a esta práctica explicaría que, actualmente, haya tantas personas con dificultad para sostener la atención, planificar, organizar, prospectar, tomar decisiones, resolver conflictos, autorregular su conducta, sus emociones, su aprendizaje, etc. ${ }^{2526}$

Estas carencias, sin duda, se verán reflejadas en el desempeño posterior de niños y jóvenes cuando «deban» evidenciar el progreso en sus diferentes competencias. En este particular, las propias pruebas PISA (Program for International Student Assessment en inglés; Programa Internacional para la Evaluación de los Alumnos, en castellano), de las que el sistema educativo costarricense ha participado desde el 2009, refieren que estas se enfocan en evaluar competencias y consideran el conocimiento en diferentes contextos de información. Es decir, evalúan el nivel alcanzado por estudiantes en cuanto a conocimientos y capacidades esenciales para participar en la sociedad en el momento de cumplir 15 años. Una característica de la prueba PISA es que lleva a cabo una evaluación basada en problemas contextualizados e intenta ir más allá de los problemas que típicamente se plantean en las aulas. Así, para PISA, la evaluación no debe centrarse únicamente en lo que aprenden y conocen los estudiantes, sino en cómo pueden utilizar lo que aprendieron en nuevos contextos. PISA evalúa competencias en lectura, matemáticas y ciencias, pues considera a estas disciplinas como primordiales en la educación de todo estudiante y en su desempeño en el diario vivir ${ }^{27}$. Los resultados para Costa Rica, en el 2015, según el promedio establecido por la OCDE con un indicador de 493, reporta que en las áreas mencionadas se obtuvieron 427, 400 y 420 puntos, respectivamente.

En las tres áreas, Costa Rica estuvo muy por debajo del promedio y matemáticas fue el más bajo de todos, con una nota en el indicador de 400. Si bien estas pruebas son referenciales, se deben examinar cuidadosamente con el fin de que no sirvan únicamente para medir el aprendizaje sino, y primordialmente, para promover mejoras en los procesos de enseñanza-aprendizaje. La situación se agrava cuando los resultados de estas pruebas son recibidos en el contexto nacional como un sólido referente de calidad de la educación, sin profundizar en la naturaleza misma de estas. Cuando menos, se debiera preguntar si la construcción de los aprendizajes que están siendo medidos ocurre en un contexto o descontextualizadamente; si quienes los facilitan están tomando en consideración las distintas etapas del aprendizaje (código, almacenamiento, recuperación y transferencia) o si se consideran en función de simples ingresos y salidas de datos aislados.

Ahora bien, «(...) el futuro no se explica necesariamente solo por el pasado» ${ }^{28}$, los esfuerzos de todos los modelos se ven descontextualizados per se al emerger de afuera hacia adentro y, con frecuencia, de manera acrítica. De ahí que cobre especial relevancia que la gestión haga valer la oportunidad que le deviene de su ente rector,

\footnotetext{
${ }^{25}$ Julio Cesar Flores Lazaro y Feggy Ostrosky-Solis, «Neuropsicología de lóbulos frontales, funciones ejecutivas y conducta humana», Revista Neuropsicología, Neuropsiquiatría y Neurociencias 8 (2008): 47-58.

${ }^{26}$ Azucena Lozano Gutiérrez y Feggy Ostrosky Solís, Desarrollo de las Funciones Ejecutivas y de la Corteza Prefrontal, Revista Neuropsicología, Neuropsiquiatría y Neurociencias 11, n. ${ }^{\circ}$ (2011), 159172.

${ }^{27}$ Dirección de Gestión y Evaluación de la Calidad, «Qué es PISA», Ministerio de Educación Pública, acceso: 31 de agosto de 2021, https://dgec.mep.go.cr/deac/pisa/que-es-pisa

${ }^{28}$ Cassasus, Problemas de la..., 104.
} 
de atreverse a reinventar el rumbo, a desandar los pasos, a desaprender, a aprender a aprender, como lo sugiere la reingeniería. En este mismo sentido, la gestión de calidad total propone que exista una identificación con los usuarios al punto de suplir sus necesidades con altos estándares de calidad, pero de manera continua.

No son suficientes los cambios por el simple hecho de cambiar, todo cambio debe estar bien fundamentado, incluso, puede ser más cualitativo que cuantitativo o un complemento de ambos. En algunos contextos bastará con optimizar los recursos existentes, en otros significará replantearse por completo el norte. Sin embargo, con el propósito de reducir la incertidumbre que implica cualquier cambio, muchas veces, se ha vuelto tendencia acudir a la «receta». Época tras época, estas prácticas de apostarle a la inmediatez y a la receta le han traído múltiples repercusiones al ámbito educativo, las facturas, según se lee en el Séptimo Informe del Estado de la Educación, no son pocas.

La teoría del procesamiento de la información, con particular énfasis en el desarrollo cognitivo, explica que la memoria permite codificar, almacenar y recuperar información, pasando por diferentes vías. Ahora se sabe que la memoria tiene una función adaptativa, porque permite resolver problemas a partir de las experiencias previas y no solo recordar información. Por eso el conocimiento previo es el punto de partida para construir aprendizaje nuevo, de ahí que los docentes propician la evocación de lo que el estudiante conoce cuando presenta un contenido nuevo. «Una buena estrategia en el aula es la búsqueda de ideas previas, rastrear el bagaje con el que llegan los alumnos e intentar establecer dichas asociaciones», sugiere en una de sus publicaciones la Asociación Educar para el Desarrollo, de Argentina ${ }^{29}$. Para que ese procesamiento de la información sea significativo ${ }^{30}$, requiere pasar por tres «filtros»:

a) la memoria sensorial (dura segundos o incluso menos cuando no se presenta información disociada del conocimiento previo del estudiante, meros datos aislados, simplemente se diluye),

b) la memoria de trabajo u operativa (dura horas y solo guarda de cuatro a siete pedazos de información) y, finalmente,

c) la memoria a largo plazo (la de duración ilimitada). Cabe resaltar que la memoria es un tema complejo, en constante estudio, los aportes podrían variar incluso de autor en autor, para efectos de este artículo se remite a estas tres sin ahondar en los tipos de cada una.

Además, de acuerdo con lo que explica Mc Clean ${ }^{31}$, a la hora de presentar un contenido a los estudiantes, se debe comprender que hay una especie de peajes que conviven en cada quien (teoría del cerebro triuno):

a) un sistema que sufre de «neofobia»; fue, evolutivamente hablando, «diseñado» para defenderse, atacar o escapar; es instintivo, inconsciente;

\footnotetext{
${ }^{29}$ Mariela Vestfrid, «Estudiar de memoria o con memoria», acceso: 31 de agosto de 2021, https://asociacioneducar.com/estudiar-de-memoria

${ }^{30}$ David Presti, Foundational Concepts in Neuroscience: A Brain-Mind Odyssey (New York: W. W. Norton \& Company, 2015).

${ }^{31}$ Lucas Raspall, Neurociencias para educadores (Rosario: Homo Sapiens Ediciones, 2017).
} 
b) el sistema emocional (visceral y no verbal), también inconsciente; evolutivamente hablando, vino a mejorar el anterior, pero oscila, según Raspall, entre «...evitación del daño y búsqueda de placer».

Para pasar el primer filtro hay que comprar un pase que diga «esto es esencial para mi vida ${ }^{32}$, lo cual remite a una de las tres funciones del sistema nervioso: la supervivencia. El sistema emocional, como se vio, también vino con una función evolutiva muy importante y su pase, a diferencia del anterior, deberá tener imprimidos algunos de los siguientes términos: «emoción», «novedad», «asombro». No se puede perder de vista, eso sí, que tal y como lo explican los neurocientíficos, lo que hoy es novedoso para este sistema, mañana no lo será.

Un tercer sistema, el racional (neocorteza), considerado el lujo de la evolución, está asociado con las funciones ejecutivas ${ }^{33}$. A diferencia de los dos anteriores, es consciente. Esta contiene a la corteza prefrontal (ubicada en la porción anterior de lóbulo frontal), involucrada con las Funciones Ejecutivas (FE), es decir, con actividades mentales complejas necesarias para atender una tarea, la toma de decisiones, la capacidad de inhibir o emprender una acción, de planificar, organizar y supervisar las metas, regular el propio pensamiento y aprendizaje (metacognición), diálogo interno, memoria de trabajo (mantener en la mente información requerida para tareas presentes o futuras), previsión (prever las consecuencias), retrospección (recordar experiencias pasadas), etc. Pero también tiene su particularidad: consume una buena dosis de gasto energético, puede sufrir apagones si se sobreexige y requiere entrenamiento durante 20 y 30 años a lo largo de la vida, dependiendo de si se es varón o mujer, respectivamente (estos datos varían de autor en autor).

¿De qué modo se aprovecharía este insumo en el quehacer pedagógico? Para comenzar, ahora se sabe que el cerebro aprende de manera integral, por eso las nuevas propuestas curriculares instan a crear contextos situacionales, a dejar de enseñar contenidos separadamente ${ }^{34}$. Los estudios de neurociencias reafirman y consolidan caminos trazados por Vigotsky y Luria con respecto a la relevancia que tiene el trabajo colaborativo (ZDP): se aprende mejor si un compañero experto explica y esos roles son intercambiables (el que hoy explica algo podría recibir en otro momento una explicación). Ahora bien, no se trata de asignarle al estudiante el rol que solamente puede ejercer el docente, sino de crear las condiciones propicias para que, conjuntamente, los estudiantes se apropien de las estrategias que les permitirán autorregular su aprendizaje. En este sentido, el docente es un promotor de estrategias. Conocer mejor la constitución neurobiológica permite comprender por qué se advierte que el aprendizaje es significativo o no es aprendizaje, esto quiere decir que los estudiantes recordarán el contenido relevante para sus vidas (sistema instintivo), lo que lleve una buena dosis de novedad, emoción y asombro (sistema emocional). No menos importante es reconocer que el aprendizaje y el miedo son incompatibles $^{35}$, que el error es la mejor fuente de aprendizaje.

\footnotetext{
32 Presti, Foundational Concepts in...

${ }^{33}$ Azucena Lozano Gutiérrez y Feggy Ostrosky, «Desarrollo de las funciones ejecutivas y de la corteza prefrontal», Revista Neuropsicología, Neuropsiquiatría y Neurociencias $11, \mathrm{n} .^{\circ} 1$ (2011).

${ }^{34}$ María Antonieta Fisher, «La importancia de las neurociencias en la educación: ¿Qué nos dicen las neurociencias al respecto? ¿Qué propuestas nos ofrecen?», acceso: 31 de agosto de 2021, https://www.redem.org/la-importancia-de-las-neurociencias-en-la-educacion-que-nos-dicen-lasneurociencias-al-respecto-que-propuestas-nos-ofrecen/

${ }_{35}$ María Caballero, Neuropsicoeducación de profesores y para profesores (Madrid: Pirámide, 2017).
} 
En esta afirmación podría detenerse un poco más; el miedo, si bien tiene una función adaptativa, trae consigo la liberación de sustancias que, en altas dosis, terminan por deteriorar incluso la memoria de trabajo (cortisol, glutamato, adrenalina). El reto estriba en que quienes han sido formados en un sistema centrado en el resultado como sinónimo de éxito, conciban que una propuesta curricular pueda ser más efectiva si se enfoca en el estudiante como protagonista de un proceso que le permitirá desarrollar habilidades, en lugar de convertirlo en un receptor pasivo de contenidos que, además, no encuentra relevantes para su vida. Entonces, cobran sentido principios como «se recuerda mejor lo que se explica con sus propias palabras».

Ahora bien, de acuerdo con la experiencia personal, contrario a lo que se esperaría, se ha estado sembrando "aprendizaje» en un caldo de cultivo bastante cargado de ansiedad por el error. Surgen las preguntas obligadas: ¿En qué le han abonado los entornos educativos "controlados» desde afuera y la penalización del error a la calidad de la educación? ¿Cuál es el concepto de inteligencia que ha regido el sistema educativo? ¿Sigue este estrechamente vinculado al resultado numérico? ¿Qué relación hay entre inteligencia y toma de decisiones?

Entonces, si bien se dan pasos firmes hacia la transición de un modelo centrado en el «número» a otro más enfocado en la persona, como afirma Carreño, se ha formado a personas que demandan seguir rindiendo culto a la nota $\mathrm{y}$, con ella, a la fragmentación del proceso. Además, reducen el concepto de inteligencia al acto de engullir contenidos, siendo que neurocientíficos como Marina, entre otros, orientan más a una acepción de inteligencia asociada a la capacidad para tomar decisiones, resolver conflictos, tener un cerebro ejecutivo que gobierna el sistema emocional e instintivo y no a la inversa ${ }^{36}$. Las personas consideran que son más leyes las que se necesitan para acabar con la delincuencia y con los desafortunados incidentes de violencia que acaparan las secciones de sucesos ¿Serán más leyes sinónimo de que se tiene la capacidad de autorregularse? ¿No será eso perpetuar la regulación externa de la conducta y emociones?

Respuestas a estas y otras interrogantes deben reflexionarse y abordarse con acuciosidad si la intención de las autoridades gubernamentales y demás actores involucrados realmente aspiran a seguir construyendo una propuesta de educación que responda a las necesidades del siglo XXI.

Sin duda, neurociencias no es un lujo ni una moda. El resultado de los estudios acerca de cómo aprende el cerebro permea la educación, son conocimientos que nadie que gestione la educación con responsabilidad puede eludir ni ignorar, es de alto impacto en la educación y en la construcción de cualquier propuesta educativa para el futuro.

Al respecto, hay que decir que en Costa Rica, las últimas reformas curriculares contemplan el desarrollo de funciones ejecutivas y el entrenamiento de la conciencia fonológica desde el nivel de preescolar. El primero es un predictor de la conducta responsable (las materias son el insumo o excusa para desarrollarlas); el segundo, el predictor del éxito del proceso lectoescritor. Se hace referencia a habilidades que, de acuerdo con los teóricos, se originan en el plano familiar y de este trascienden al de la educación formal ¿Están las familias conscientes de la relevancia que tienen para el desarrollo de tales habilidades? La dinámica familiar ha cambiado, la agenda

\footnotetext{
${ }^{36}$ Jose Antonio Marina, video: La inteligencia ejecutiva, https://www.youtube.com/watch?v=cHg52tn7uoA
} 
laboral ha desplazado un poco el plano de las interrelaciones, entonces ¿Qué papel juega la escuela en el desarrollo de estas habilidades?

El Programa de Estudio de Educación Preescolar ${ }^{37}$-que se adscribe al modelo pedagógico desarrollista, cuyo fin último es que la persona se construya gradual y progresivamente en un ser integral- se nutre, entre otros, de los avances de la psicología infantil y de los aportes de neurociencias a la educación. No han sido pocos los esfuerzos de las últimas administraciones para revolucionar un modelo pedagógico que se perdía al desembocar en otro que giraba en torno a una nota. Conducta y pensamiento van de la mano; para Vigotsky ${ }^{38}, \ll(\ldots)$ el pensamiento se hace verbal y el habla racional».

Según el Tercer Informe del Estado de la Educación ${ }^{39}$, en los periodos 2008-2010 ya se habían renovado varias de las propuestas curriculares del sistema educativo costarricense, y entre el 2012 y el 2013 se aprobaron los nuevos programas de Matemática y Español para primer y segundo ciclo.

La primera unidad de lectoescritura del Programa de Español se titula «La conciencia fonológica: ¡Fuente de inspiración para leer y escribir!» ${ }^{40}$. Sin embargo, desarrollar estas habilidades es un proceso que implica mucho más que la sola conversión de fonemas en grafemas (conciencia fonémica). Incluye tareas de análisis (segmentar, omitir, cambiar de orden, sustituir) y de síntesis (unir, mezclar). Requiere, por ende, inversión de tiempo y creatividad en el primer año de primaria para que el estudiante se apropie del código de la lengua y lo utilice deliberadamente en el nivel de la palabra, la sílaba y el fonema. De este modo, en segundo año, podría enfocarse más a la comprensión que a la decodificación. Según Stanislas Dehaene ${ }^{41}$, «Durante el primer año de la escuela primaria, la lectura supone un inmenso trabajo de atención». Cuando el estudiante pasa a segundo año sin haber logrado la conciencia del código de la lengua en sus distintos niveles y secuencias, verá mermada su capacidad de comprensión y producción, pues saturará su atención y su memoria de trabajo invirtiendo tiempo en descifrar el código e integrar mentalmente una palabra ${ }^{42}$.

Actualmente se sabe que pensar y actuar es lo mismo para el cerebro. Aprender es posible en un marco flexible y no convencional de aprendizaje, uno que no siempre es comprendido por los padres de familia que, como se ha expuesto, se mantienen asidos a un modelo conocido por ellos, según el cual «más es mejor».

En el 2019, el biólogo y neurocientífico Hernán Aldana, en conferencia para docentes de Educación de la Universidad Estatal a Distancia (UNED) de Costa Rica, se apoyó en el trabajo del neurocientífico Radek Ptak, The dorsal frontoparietal network: A core system for emulated action ${ }^{43}$, para explicar que «la red ejecutiva frontoparietal evolucionó de una simple red de control de acción a una red que se

\footnotetext{
${ }^{37}$ Ministerio de Educación Pública, Programa de Estudio de Educación Preescolar (2014).

${ }^{38}$ Carlos Álvarez González, «La relación entre lenguaje y pensamiento de Vigotsky en el desarrollo de la psicolingüística moderna», Revista de lingüística teórica y aplicada, 48 (2), 2010, 13-32, acceso: 31 de agosto de 2021, http://www.scielo.cl/scielo.php?script=sci_arttext\&pid=S071848832010000200002

${ }^{39}$ Programa Estado de la Nación en Desarrollo Humano Sostenible, Estado de la Educación (San José: Consejo Nacional de Rectores, 2011).

${ }^{40}$ Ministerio de Educación Pública, Programa de Español I Ciclo (2013), 67.

${ }^{41}$ Stanislas, Aprender a leer..., 54-59.

42 Ibíd., 59.

${ }^{43}$ Radek P., Armin S., Julia F., The Dorsal Frontoparietal Network: A score system for emulated action. Division of Neurorehabilitation (Geneva, 2017).
} 
encarga de las funciones cognitivas». Crear condiciones aptas para la construcción de aprendizajes efectivos pasa primero por la deconstrucción de roles, maneras de hacer y de concebir el acto de educar, que no logra trascender la sola dimensión académica. Difícilmente se propiciarán aprendizajes significativos sin involucrar a la persona, su bagaje personal, familiar, cultural y social. En palabras de Aldana, si se desea calar en la persona, el cuerpo va primero.

Se considera simplista aducir que involucrar el cuerpo únicamente hace alusión al movimiento (punto de partida para recordar mejor). Sin duda, también se hace referencia a la UCCMA (unidad cerebro-cuerpo-mente-ambiente). Existe una fina línea entre el currículo centrado en el docente (el planeamiento adecuado, las prácticas estructuradas y dosificadas, por citar algunos) y el currículo centrado en el estudiante (sus intereses, sus preguntas, sus sentires, sus inquietudes, preocupaciones, avideces, etc.). Esto abarca la inmersión en sus mundos: un conocimiento $^{44}$ sobre los cambios que sufren los estudiantes adolescentes, para quienes construirse acapara buena parte de su tiempo, el ciclo del sueño; los periodos atencionales de los niños (15 a 20 minutos) según sea el contexto: virtual o presencial, etc. Lo que no se repasa, tal y como apunta las neurociencias, se olvida. Un contenido debe repasarse al menos 24 veces. La motivación intrínseca es la duradera, porque se nutre de la tarea misma.

De lo expuesto se desprende la relevancia que tiene para la escuela el crear puentes de formación con las familias. El divorcio entre ambos actores del currículo no es una opción. Le corresponde a la escuela justificar ante las familias la urgencia de deconstruirse: que las lecciones comiencen a las 8:00 a. m. y no se extiendan más del mediodía (duración de la atención ejecutiva), que las clases estén estructuradas en sesiones de 30 minutos y no de 40, que la dinámica de la clase no gire en torno al docente sino al estudiante, que se dediquen tiempo, recursos y esfuerzos al desarrollo de las etapas del aprendizaje (codificación, mantenimiento, recuperación), que la actividad física en el aula no es una pérdida de tiempo y que favorece los procesos de mielinización (favorecen las conexiones neuronales), que los espacios para que el estudiante se conozca a sí mismo (comprenda cómo aprende, cómo comprende, cómo recuerda, etc.) son imprescindibles, que la motivación intrínseca (alimentada por la curiosidad e interés propios) es la duradera y no la extrínseca (supeditada a signos externos, premios, reconocimiento, castigo), que el reto de la actual generación no es dominar la información que sobreabunda, sino saberla gestionar (un pensamiento flexible emerge de la construcción de criterio), que es necesario un proceso de evaluación continua y en doble vía (valorar el resultado de este y no un contenido), uno que retroalimente al estudiante y al docente.

Sigue siendo el vínculo que caracteriza a la humanidad lo que justifica que en la clase esté una persona y no una máquina: la serotonina generada por el respeto, la dopamina por la sensación de logro, la endorfina por la sensación de que puedo aprender independientemente de mis errores, la oxitocina que se desprende de la mirada ojo a ojo, del cálido roce de reconocimiento de la persona, sin que para ello medie un resultado.

Indudablemente, las neurociencias han abierto, a través del conocimiento del cerebro, una ruta intransitada hacia las bases neurológicas del aprendizaje.

\footnotetext{
${ }^{44}$ María Encarnación Peña Bonilla, «Neurociencias y educación van juntas a las aulas», acceso: 31 de agosto de 2021, https://www.ucr.ac.cr/noticias/2017/07/26/neurociencias-y-educacion-van-juntas-alas-aulas.html
} 
Acompañar a una persona -reconociéndola como una unidad, como un todoposibilita habilitar más y mejores caminos de acceso hacia el aprendizaje y, de paso, la resignificación del aprendizaje mismo y, en general, del acto de educar. Lo cierto es que, si bien son los neurocientíficos los que proveen sólidos conocimientos para la práctica pedagógica, son los docentes los que pueden cristalizar esos aportes en el aula, quitándole a esta la connotación actual limitada a una «sala donde se dan clases» y dotándola de una más dinámica, abierta, emergente (vinculada con la vida en la que se inscribe ese estudiante y ese docente).

En este artículo solo se han plasmado algunas pinceladas de lo que grandes proponentes y referentes plantean en torno de temáticas que se entretejen, como son la calidad de la educación y la gestión educativa: Cassasus (2007), Frith y Blakemore (2011), Raspall (2017), Marina (2014), Lozano \& Ostrosky (s.f), Presti, Flores \& Ostrsky (s.f), Séptimo Informe del Estado de la Educación (2019), D’ Antoni (2015), Retana (2016), Garnier (2011), Radek Ptak, Armin Schnider, Julia Fellrath (2017), entre otros.

\section{A manera de síntesis}

Finalmente, se anotan algunas de las conclusiones que se desprenden de las ideas planteadas:

- La educación del siglo XXI debe servir al desarrollo integral de la persona, debe ser abarcante: una educación para todos. Esto implica colocar los objetivos en favor del despliegue de habilidades. Conlleva implementar metodologías multisensoriales, conocer y comprender mejor cómo aprende el cerebro humano y sin dejar de lado el bagaje que cada persona trae consigo al centro educativo, primordialmente, que ante todo se es persona, para generar pedagogías y didácticas congruentes.

- El sistema de selección del personal docente por parte de las autoridades gubernamentales (MEP) debe trascender la valoración del acervo profesional, involucra otras variables: en un mundo tan cambiante, la persona debe saberse un eterno aprendiz, disfrutar del trabajo con otras personas, saber gestionar sus propias emociones y realizar lectura adecuada de las del otro, tener un pensamiento flexible, etc.

- El acto de educar y el de gestión deben pensarse y repensarse si verdaderamente se quiere innovar y mejorar la calidad del modelo educativo.

- Las generaciones del siglo XXI no pueden gestionarse más a través de prácticas propias de la década de 1980.

- El quehacer pedagógico de quienes facilitan el proceso de enseñanza-aprendizaje de las generaciones del siglo XXI, bien acompañado, es un puente para que los aportes de neurociencias crucen al aula y florezcan.

- El aprendizaje es significativo o no es aprendizaje. El error no debe ser penalizado, sino aprovechado como fuente de aprendizaje. Ya se sabe que el miedo y el aprendizaje son incompatibles, el progreso de los estudiantes debe procurarse enfatizando en el «cómo», no en la siembra de miedos ni en el anuncio de castigos, aniquilantes por excelencia de la motivación intrínseca.

- La evaluación tradicional fragmenta el proceso para concentrarse en el porcentaje y de lo que se trata es de valorar el proceso y calificar el resultado de ese proceso. No necesariamente se trata de desaparecer el número (la nota), sino la manera como 
se evalúa. La evaluación debe servir al aprendizaje y no solamente evaluar los aprendizajes, ese es el propósito de las pruebas formativas, ser fuente de retroalimentación para docente y estudiante, por ende, la evaluación va y viene, es un camino de doble vía.

- La diferencia entre educar y enseñar es que el estudiante, cuando se educa, vale más que los objetivos. La persona ha de ir primero si se desea que eso regrese a la sociedad, en el sistema social que sale de las aulas. El currículo es el medio para que la persona se desarrolle de manera integral y no el fin.

- Según José Antonio Mariana ${ }^{45}$, el concepto de inteligencia ha variado: ahora está asociada a toma de decisiones, porque el control, el verdadero, está dentro de cada uno. $\mathrm{Su}$ falta de entrenamiento deriva en personalidades erráticas, dificultades de atención, de control de impulsos, etc.

- Las investigaciones neurocientíficas son vastas, dotan a la educación de herramientas poderosas para acompañar de manera más efectiva al estudiante: neurocientíficos estudian el cerebro humano y los docentes concretan en el aula el resultado de esas investigaciones gracias al acercamiento que han tenido recientemente unos con otros. Eso ya no es un lujo ni una moda, es un acto de amor.

- Los aportes de neurociencias también se pueden extrapolar a la gestión. Para Heidegger, «(...) en toda acción hay una emoción». La persona que trabaja desarrollando el talento humano debe, ante todo, ser capaz de gestionar adecuadamente la comunicación y comprender las emociones, lo cual implica tener claridad en que ambas son el resultado de distintas vías: la primera es racional, consciente y verbal; la segunda es inconsciente y visceral ${ }^{46}$.

- Incluir rutinas y procesos flexibles. Las rutinas son importantes, pero no deben ser inflexibles, la novedad y el asombro son relevantes.

- Privilegiar el esfuerzo individual y el trabajo cooperativo, el trabajo guiado y el trabajo por proyectos (para favorecer la flexibilidad). La corteza prefrontal está conectada con la amígdala y con el sistema de motivación y gratificación de la dopamina.

- Las programaciones deben ir de lo simple y concreto a lo complejo y abstracto.

- Hay que saber lo que se le puede «pedir» (lo que se puede esperar también) a un niño y a un joven, dado que el «control inhibitorio» se termina de desarrollar después de la adolescencia ${ }^{47}$. Si todavía no se cuenta con la capacidad de detener el impulso (la corteza prefrontal es la que permite evaluar los contextos), el estudiante todavía no sabrá elegir adecuadamente.

- Con respecto a la metacognición (una de las funciones ejecutivas más complejas), para facilitar el desarrollo de estrategias metacognitivas, el docente debe convertirse en alguien que incentiva la generación de estrategias, las dirige, pero no se apropia de ellas (además de explicar le permite al estudiante reconstruir lo aprendido con sus propias palabras).

\footnotetext{
${ }^{45}$ José Antonio Marina, La inteligencia Ejecutiva: lo que los padres y docentes deben saber (Barcelona, España: Ariel, 2012).

${ }^{46}$ Cassasus, Problemas de la ..., 109.

${ }^{47}$ Lucas Raspall, Neurociencias para educadores (Rosario: Homo Sapiens Ediciones, 2017).
} 
- La persona docente ha de propiciar que su estudiante vuelva la mirada hacia su proceso interno. Al hacerlo, deberá romper con la mecanicidad; es decir, promoverá procesos conscientes y sistematizados. Será capaz de conducirlo al automonitoreo del proceso y del resultado respectivamente a través de interrogantes tales como «¿Qué estoy haciendo?» «¿Por qué lo hago?» «¿Cómo se hace?» «¿Cómo lo estoy haciendo?» «¿Qué tal lo hice?» «¿Qué dificultades tuve?» «¿Cómo puedo mejorar?» «¿Me gustó hacerlo?» «¿Cómo me sentí?» Sin metacognición no es posible hablar de autorregulación de la conducta, de las emociones y del aprendizaje.

Formato de citación según APA

Anchía-Angulo, L. y Brizuela-Gutiérrez, T. (2021). La educación en el siglo XXI: una mirada incipiente desde las neurociencias al quehacer pedagógico. Revista Espiga, 20 (42), 17-36.

Formato de citación según Chicago-Deusto

Anchía-Angulo, Lía y Brizuela-Gutiérrez, Tobías. «La educación en el siglo XXI: una mirada incipiente desde las neurociencias al quehacer pedagógico». Revista Espiga 20, n. ${ }^{\circ} 42$ (setiembre, 2021): 17-36. 


\section{Referencias}

Álvarez González, Carlos. «La relación entre lenguaje y pensamiento de Vigotsky en el desarrollo de la psicolingüística moderna». Revista de lingüistica teórica y aplicada 48 (2), 2010, 13-32. Acceso: 31 de agosto de 2021. http://www.scielo.cl/scielo.php?script=sci_arttext\&pid=S071848832010000200002

Blakemore, Sarah y Frith Uta. Cómo aprende el cerebro. Las claves para la educación. Barcelona: Editorial Ariel, 2011.

Caballero, María. Neuropsicoeducación de profesores y para profesores. Madrid: Pirámide, 2017.

Cassasus, Juan. Problemas de la gestión en América Latina. La tensión entre los paradigmas A y B. Revista Brasilia, 19, n. ${ }^{\circ} 75$ (49-69), 2002.

Consejo Nacional de Rectores. Séptimo Informe del Estado de la Nación.

D’ Antoni, Maurizia. «Carmen Lyra: una Pedagogía acallada en Costa Rica y sus vinculaciones teóricas». Ponencia del $3^{\text {er }}$ Congreso Internacional de Investigación Educativa. Educación y Globalización. Instituto de Investigación en Educación, Universidad de Costa Rica, 2015.

Fisher, María Antonieta. «La importancia de las neurociencias en la educación: ¿Qué nos dicen las Neurociencias al respecto? ¿Qué propuestas nos ofrecen?». Acceso: 31 de agosto de 2021. https://www.redem.org/laimportancia-de-las-neurociencias-en-la-educacion-que-nos-dicen-lasneurociencias-al-respecto-que-propuestas-nos-ofrecen/

Flores, Julio Flores y Feggy Ostrosky. «Neuropsicología de lóbulos frontales, funciones ejecutivas y conducta humana». Revista Neuropsicología, Neuropsiquiatría y Neurociencias 8 (2008): 47-58.

Flores, Julio Flores y Feggy Ostrosky. «Desarrollo de las funciones ejecutivas y de la corteza prefrontal». Revista Neuropsicología, Neuropsiquiatría y Neurociencias 11, n. $^{\circ} 1$ (2011): 159-172.

Garnier, Leonardo. «¿Mejor, no se arriesgue?». Acceso: 31 de agosto de 2021. http://leonardogarnier.com/articles/mep-subversivo/mejor-no-se-arriesgue-746

Lozano Gutiérrez, Asucena y Feggy Ostrosky, L. «Desarrollo de las funciones ejecutivas y de la corteza prefrontal». Revista Neuropsicología, Neuropsiquiatría y Neurociencias 11, n. ${ }^{\circ}$ (2011): 159-172.

Marina, José Antonio. La inteligencia Ejecutiva: lo que los padres y docentes deben saber. Barcelona, España: Ariel, 2012.

Marina, José Antonio. Video: La inteligencia ejecutiva. Acceso: 31 de agosto de 2021. https://www.youtube.com/watch?v $=\mathrm{cHg} 52 \operatorname{tn} 7 \mathrm{uoA}$ 
Marina, José Antonio. «Neurociencia y educación. Participación Educativa». Revista del Consejo Escolar del Estado 1, n. ${ }^{\circ} 1$ (2012): 1-13.

Ministerio de Educación Pública. Documento Resultados Prueba Operativa de Control. Acceso: 31 de agosto de 2021. PISA | Dirección de Gestión y Evaluación de la Calidad (mep.go.cr)

Ministerio de Educación Pública. Programa Oficial de estudios para Preescolar. MEP, 11.

Ortiz Ocaña, Alexander. Neuroeducación. ¿Cómo aprenden los estudiantes y cómo deberían enseñar los docentes? Bogotá: Ediciones de la U, 2015.

Peña Bonilla, María Encarnación. «Neurociencias y Educación van juntas a las aulas». Acceso: 31 de agosto de 2021.

https://www.ucr.ac.cr/noticias/2017/07/26/neurociencias-y-educacion-vanjuntas-a-las-aulas.html

Pérez, César. «La educación es alma del desarrollo de los pueblos, dijo Rigoberta Menchú». La voz del sanidnismo, 16 de febrero de 2011. Acceso: 31 de agosto de 2021. https://www.lavozdelsandinismo.com/nicaragua/2011-0216/la-educacion-es-alma-del-desarrollo-de-los-pueblos-dijo-rigobertomenchu/

Presti, David E. Foundational Concepts in Neuroscience: A Brain-Mind Odyssey. New York: W. W. Norton \& Company, 2015.

Programa Estado de la Nación en Desarrollo Humano Sostenible. Estado de la Educación. San José: Consejo Nacional de Rectores, 2011.

Programa Estado de la Nación en Desarrollo Humano Sostenible. Resumen Séptimo Informe Estado de la Educación. San José: Mastelirtto, 2019.

Radek P., Armin S., Julia F. The Dorsal Frontoparietal Network: A score system for emulated action. Division of Neurorehabilitation. Geneva, 2017.

Raspall, Lucas. Neurociencias para educadores. Rosario: Homo Sapiens Ediciones, 2017.

Retana, German. Entrevista y comunicación personal, 26 de abril del 2016.

Stanislas, Dehaene. Aprender a leer. De las ciencias cognitivas al aula. Buenos Aires: Siglo Veintiuno, 2015.

Vesfrid, Mariela. «Estudiar de memoria o con memoria». Acceso: 31 de agosto de 2021. https://asociacioneducar.com/estudiar-de-memoria 